\title{
Author Correction: Trends in kinase drug discovery: targets, indications and inhibitor design
}

\author{
Misty M. Attwood (iD, Doriano Fabbro, Aleksandr V. Sokolov, Stefan Knapp (iD and Helgi B. Schiöth
}

Correction to: Nature Reviews Drug Discovery https://doi.org/10.1038/s41573-021-00252-y, published online 5 August 2021.

Errors in the description of side effects reported for the kinase inhibitor deucravacitinib have been corrected.

https://doi.org/10.1038/s41573-021-00303-4 I Published online 2 September 2021

(c) Springer Nature Limited 2021

\section{Author Correction: PI3K inhibitors are finally coming of age}

Bart Vanhaesebroeck (1), Matthew W. D. Perry (1), Jennifer R. Brown (1), Fabrice André (1) and Klaus Okkenhaug(D)

Correction to: Nature Reviews Drug Discovery https://doi.org/10.1038/s41573-021-00209-1, published online 14 June 2021.

Some minor inaccuracies regarding the characteristics and clinical trials of alpelisib have been corrected.

https://doi.org/10.1038/s41573-021-00300-7 I Published online 1 September 2021

๑) Springer Nature Limited 2021 